\title{
Development of bite guard for wireless monitoring of bruxism using pressure-sensitive polymer
}

\author{
Jung Ho Kim ${ }^{1}$, Padraig McAuliffe ${ }^{2}$, Brian O’Connel ${ }^{2}$, Dermot Diamond ${ }^{1}$ and King Tong Lau ${ }^{1}$ \\ ${ }^{1}$ National Center for Sensor Research, Dublin City University, Republic of Ireland \\ ${ }^{2}$ Department of Dental school, Trinity College Dublin, Republic of Ireland \\ kim.lau@dcu.ie
}

\begin{abstract}
A wireless pressure sensing bite guard has been developed for monitoring the progress of bruxism (teeth grinding during sleep); as well as for protecting the teeth from damages. For sensing the grinding event effectively in restricted space and hostile environment, a pressure sensitive polymer composite which is safe for intra oral applications has been fabricated and encapsulated into a conventional bite guard. Also encapsulated was a microcontroller-based electronic circuit which was built in-house for data collection and transmission. A low power approach was configured to maximize the working life-time of the device to several months. The device can provide real-time tooth grinding profile through wireless communication. This device is anticipated to be a useful tool for understanding and treating bruxism.
\end{abstract}

Keywords - Bruxism, pressure sensitive polymer composite, wireless bite guard, tooth-grinding monitor

\section{Introduction}

\section{Bruxism}

It is estimated that about $10 \%$ of the population suffer from bruxism. This disease is a movement disorder of masticatory system that results in involuntary grinding of the teeth and the clenching of the jaw during sleep as well as wakefulness [1-3]. Tooth clenching or grinding during sleep can result in abnormal wear patterns of the occlusal surface, fractures in the teeth, morning headaches and facial muscle pain [4]. The most common method of bruxism management is based on minimizing the abrasion of tooth surfaces by wearing a bite-guard [5, 6]. Currently, there is no definitive clinical diagnostic method for assessing bruxism. Reliable, easy to use devices for longterm continuous monitoring of bruxism are not available.

\section{Bruxism detection and monitoring}

Evaluation of existing tooth wear does not provide evidence of current bruxism. Generally, bruxism diagnosis is to monitor masticatory muscle activities by using the surface Electromyography (EMG) [7-9]. However, the surface EMG signal is affected by factors such as electrode position, posture and skin resistance. In addition, it is not easy to attach multiple electrodes on the face without causing unease or disrupting sleep. An alternative way to diagnose bruxism is to measure bruxism activity directly in situ using pressure sensitive transducer. Several researchers have measured sleep bruxism activity directly using an intra-oral appliance. Nishigawa et. al. measured the bite force using straingauge transducer incorporated bite guard. This device was an analogue pressure sensor with electric wires connected out of the mouth during sleep. [10] Takeuchi et. al. proposed a pressure sensing device by using piezoelectric film-based sensor. However, the nature of piezoelectric transducer has limited the range of force it could sustain. [11] Despite the number of techniques being developed to detect bruxism, a practical method is still not available to monitor the progress of the symptom.

\section{Wearable splint for real time monitoring of bruxism}

In this investigation, we propose a wireless intra oral wearable pressure sensing device which offers continuous monitoring of suspected grinding over a time period to 
allow the diagnosis of the problem.

The concept envisages a carbon black-polymer composite based pressure sensor integrated into a normal prescription bite-guard. This type of pressure-sensitive composite has been investigated as force sensor [12-15]. The main advantages of the proposed sensor are: safe for in-vivo applications, easy to fabricate, chemically and physically stable with high and tunable sensitivity. The proposed device will have all electronic components encapsulated into the body of the bite-guard which will detect and wirelessly transmit in real time the grinding events to a computer. It is envisages that the device will identify patients with an active problem; monitor the progress of the symptom and to access the effectiveness of the treatment.

Due to the remarkable recent development in IC technologies that provides small size, low power microcontrollers and RF communication technologies; it has made the development of wearable sensing devices feasible $[16,17]$. The conceptual drawing of the proposed device is shown in Figure 1.

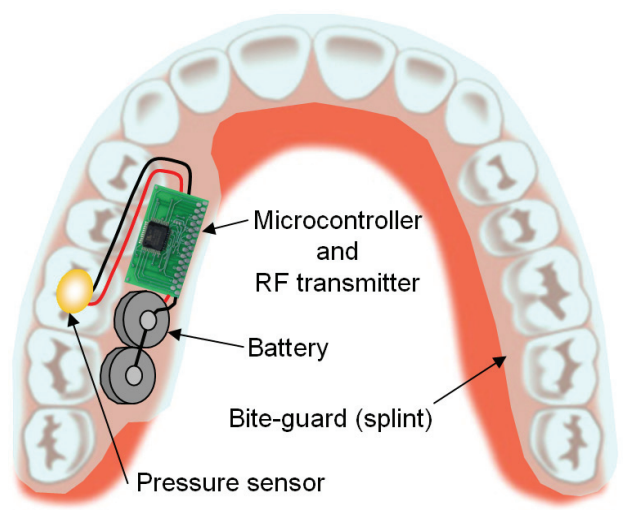

Fig. 1. Conceptual drawing of the proposed device.

A pressure sensor is incorporated inside the bite-guard as the pressure transducer. Microcontroller-based electronic circuit board including wireless module to perform essential functions including sensor control, data logging, data transmission through wireless communication and power management. A low power approach is configured to increase the working life of the device. To reduce power consumption, the device is normally set at sleep mote and would only start to transmit when a threshold value is crossed. Transmission in packets of 8 data points per second is used.

\section{Experimental}

The configuration of the proposed system used for monitoring bruxism activities is shown in Figure 2. It is composed of three modules: (1) A pressure sensor with (2) microcontroller and wireless transmitter module incorporated within a bite guard and (3) a separate RF receiver module which is connected through USB to the host computer for logging data.

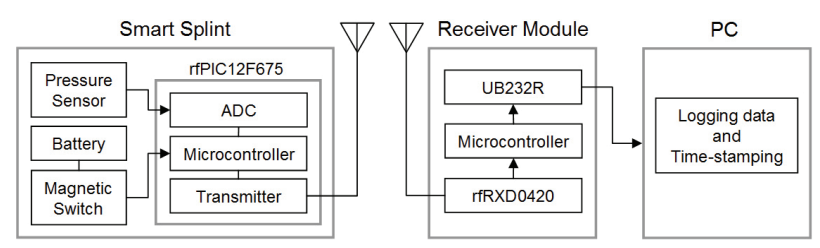

Fig. 2. System Configuration: pressure sensing bite guard with wireless transmitter, receiver module and host computer.

The size of the pressure sensing bite-guard is no bigger than conventional prescription bite-guard despite its complexity; hence the patient could wear this module without adding discomfort. The RF frequency used is $433 \mathrm{MHz}$ to avoid interference with typical household devices.

\subsection{Hardware construction}

\section{Fabrication of pressure sensor}

Conductive carbon black powder (Carbot Corp, Rozenburg, Netherlands) was ground for 5 minutes using impact milling grinder (IKA works, Inc., A11). PDMS 
(Polydimethylsiloxane: Dowcorning, Sylgard 184) prepolymer was prepared by mixing 10:1 ratio (base : curing agent) and the mixture of PDMS was degassed for 15 minutes under vacuum to remove bubbles. The mass ratio of carbon black to PDMS mixture was 17-19 wt\%.

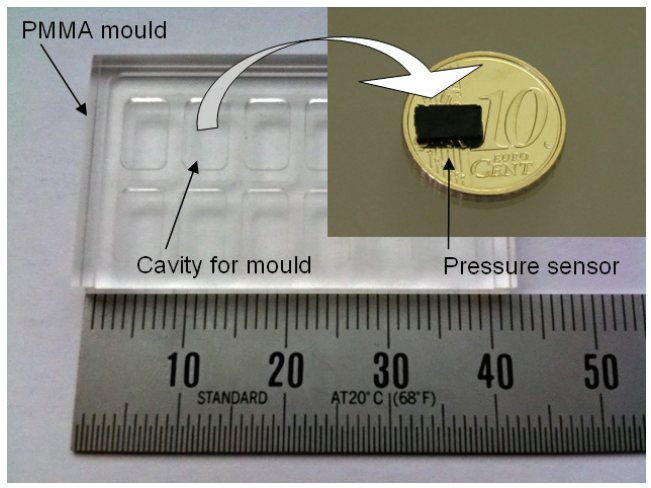

Fig. 3. Micro milled master mould and fabricated pressure sensor

The master mould for forming pressure sensor was fabricated using PMMA (Poly methyl methacrylate) plate by $\mathrm{CAD} / \mathrm{CAM}$ process and the size of sensor cavity is 7.5 $\mathrm{mm} \times 4.5 \mathrm{~mm} \times 0.6 \mathrm{~mm}$ as shown in Figure 3. After filling the mixture of carbon black and PDMS into the master mould, ultrasonic vibration was applied for 15 minutes for removal of air bubbles. Finally, the prepared mould was placed in the oven at $65^{\circ} \mathrm{C}$ for $24 \mathrm{~h}$.

\section{$\underline{\text { Electronic Circuitry }}$}

An 8 bit CMOS microcontroller rfPIC12F675 (Microchip Technology Inc.) with built-in UHF ASK/FSK transmitter was used owing to its low power consumption and compact size (20 pin SSOP package: $7.85 \mathrm{~mm}$ x $7.20 \mathrm{~mm}$ x $1.85 \mathrm{~mm}$ ). In this design, ASK (Amplitude Shift Keying) modulation is used to transmit the signal. It has a 2 digital I/O and 10 bit A/D converter with 4 analog inputs. Therefore, 4 signals can be read from different sensors and transmitted with a $433.92 \mathrm{MHz}$ carrier. In order to make the electronic circuitry small enough to be incorporated into the bite-guard, it was designed into two modules connected via flexible wirings as shown in Figure 4. The main circuit board (on the left) including microcontroller with the wireless module, the second circuit board is $\mathrm{I} / \mathrm{O}$ ports with signal conditioning function.

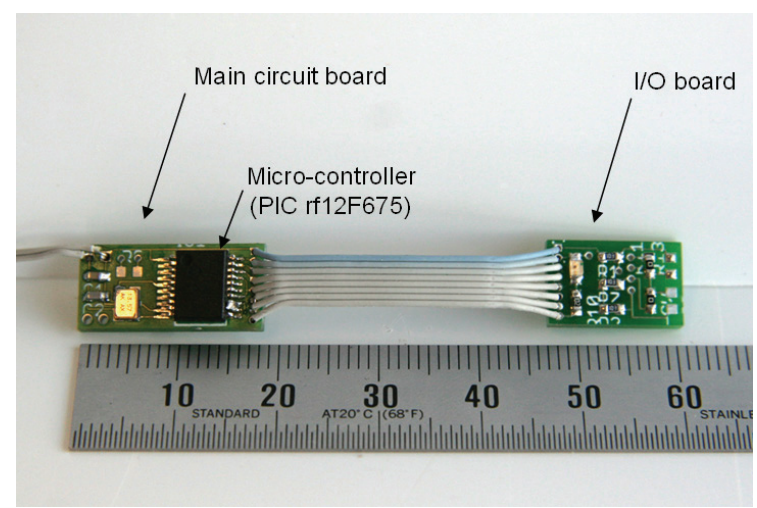

Fig. 4. Photograph of fabricated microcontroller board and $\mathrm{I} / \mathrm{O}$ board

The microcontroller regularly checks the value from the pressure sensor and transmits data packet every second when measured value crossed the predefined threshold which is decided by calibration process. Each data packet is composed of the ID number of each sensor and the $\mathrm{A} / \mathrm{D}$ value measured from the pressure sensor. Specific wireless protocol was programmed for securing data. Blinking LED on the I/O circuit board provides RF power 'ON' notification.

The receiver module was designed and fabricated using radio frequency receiver module (rfRXD420) and microcontroller (PIC12F675) from Micro Chip Inc. The microcontroller detects radio signal using interrupt routine and checks received data for errors. And the receiver module sends one byte containing sensor ID and a second byte containing data to the PC through the USB cable at 9600 baud rate and this module is powered by PC through USB 5V supply. 


\subsection{Software development}

There are three softwares in the system. The first part is bite guard microcontroller programming. Main functions of this part are $\mathrm{A} / \mathrm{D}$ conversion of measured value, making up the structure of packet using the communication protocol, comparing data with threshold value before transmitting the packet to conserve power. The second part is programming of microcontroller in the receiver module. Main functions are getting data through wireless, decoding the sensor ID and A/D value with error checking and sending data into host computer through USB cable. The last part is the program in host computer for logging and displaying incoming data with time stamping. The main screen displays A/D value of each sensor with time stamping.

\subsection{Calibration}

\section{Calibration of pressure sensor}

To investigate the performance of the fabricated carbon black-PDMS composite based sensor, the pressure sensor was calibrated with Zwick instrument. The sensor was placed in between two flat metal plates, and load was applied 5 times repeatedly at $1 \mathrm{~mm} / \mathrm{min}$ speed. The thickness of sensor used was $1.0 \mathrm{~mm}$, which was fabricated using master mould. Sensor was pressed down $0.5 \mathrm{~mm}$ ( $50 \%$ compression of thickness). Conductivity changes were recorded simultaneously by digital multimeter (Keithley, 2100) with sampling rate of $40 \mathrm{~Hz}$ as Zwick instrument presses down the pressure sensor.

\section{Calibration of pressure sensor inside a test jig with} PMMA cover

As the pressure sensor needs to be encapsulated inside the PMMA based bite-guard, it is necessary to investigate the sensor response inside a PMMA test jig to simulate the bite-guard. Experimental setup of the compression test is shown in Figure 5. The PMMA test jig with a cavity of 10 $\mathrm{mm} \times 5 \mathrm{~mm} \times 1 \mathrm{~mm}$ was fabricated using micro milling machine. PMMA covers with various thicknesses from 0.8-1.4 mm were tested. Copper tapes were attached on the bottom of the cavity and the underside of the PMMA cover as contacts where the sensor was sandwiched. A press head of $4 \mathrm{~mm}$ diameter $\mathrm{x} 1 \mathrm{~mm}$ height was also machined as the compression point. There are 4 alignment pins on test jig for inducing only longitudinal movement.
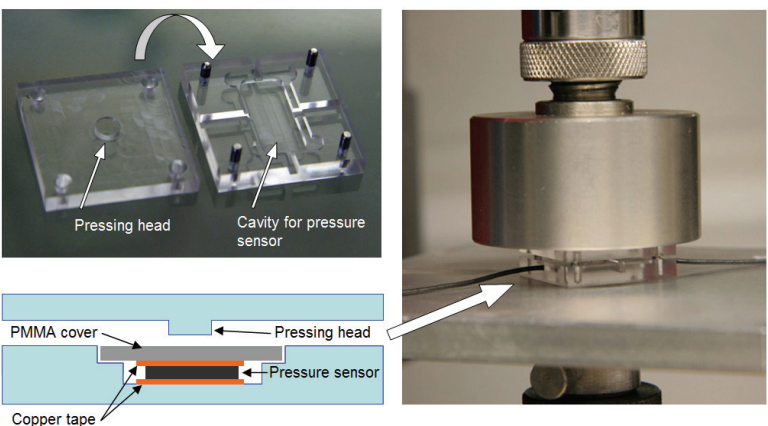

Fig. 5. Compression test for measuring the conductivity of pressure sensor using Zwick.

\section{$\underline{\text { Evaluation of pressure sensing bite guard }}$}

The finished pressure sensing bite guard prototype has microcontroller circuit board with wireless transmitter module and 2 button batteries (Silver oxide battery, SR43) integrated as shown in Figure 6. The pressure sensors were incorporated into two cavities fabricated on both sides of the molar teeth area of the bite guard. The thickness of pressure sensor and acrylic cover was $0.6 \mathrm{~mm}$ and $1.0 \mathrm{~mm}$ respectively. The integrated bite guard was then calibrated with compression test using Zwick instrument. Known load was applied 5 times repeatedly at $1 \mathrm{~mm} / \mathrm{min}$ speed. The maximum applied force for compression test was $200 \mathrm{~N}$. 


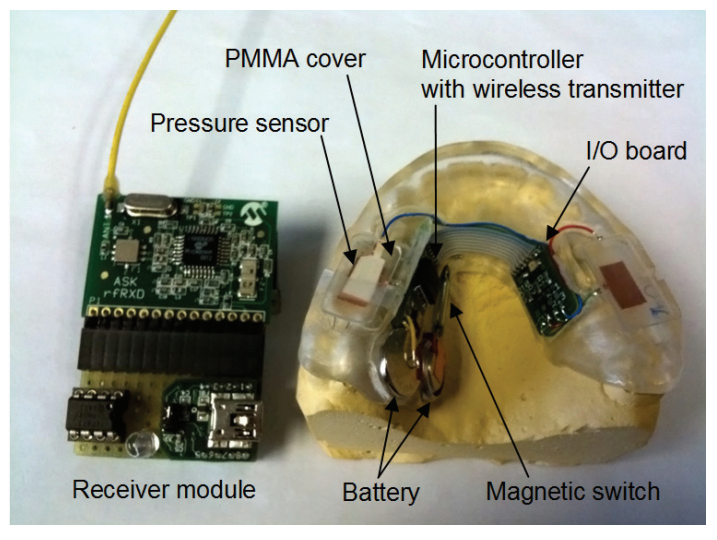

Fig. 6. Microcontroller circuit boards with pressure sensor are integrated into hard acrylic bite guard

\section{Results and discussion}

\section{Calibration of the carbon-polymer composite sensor}

The experimental results of the bare pressure sensor are shown in Figure 7. The range of resistance caused by compression test was between $67.3 \mathrm{kOhm}$ and $1.6 \mathrm{kOhm}$ when applied force was increased from $0 \mathrm{~N}$ to $61.8 \mathrm{~N}$. These results have shown that the pressure sensor gave good sensitivity over the applied force range.

It was observed that there was a slight decrease in baseline resistance on repeated compression. The reason for the observation was that the thickness of pressure sensor was not fully recovered during the consecutive loading-unloading test, therefore the pressure between the sensor and the copper contact decreased before reaching equilibrium as shown in Figure 7(a). This problem can be easily solved by pre-maturing the sensor, and incorporating the pressure sensor into the bite-guard at a pre-compressed state. Figure 7(b) presents the typical resistance change of the sensor and the simultaneous force/displacement curve. Figure 7(b) top, shows that as the slope of resistance became flatten rapidly after $250 \mathrm{sec}$ during the compression, the effective sensing limit of applied force was $25.5 \mathrm{~N}$.
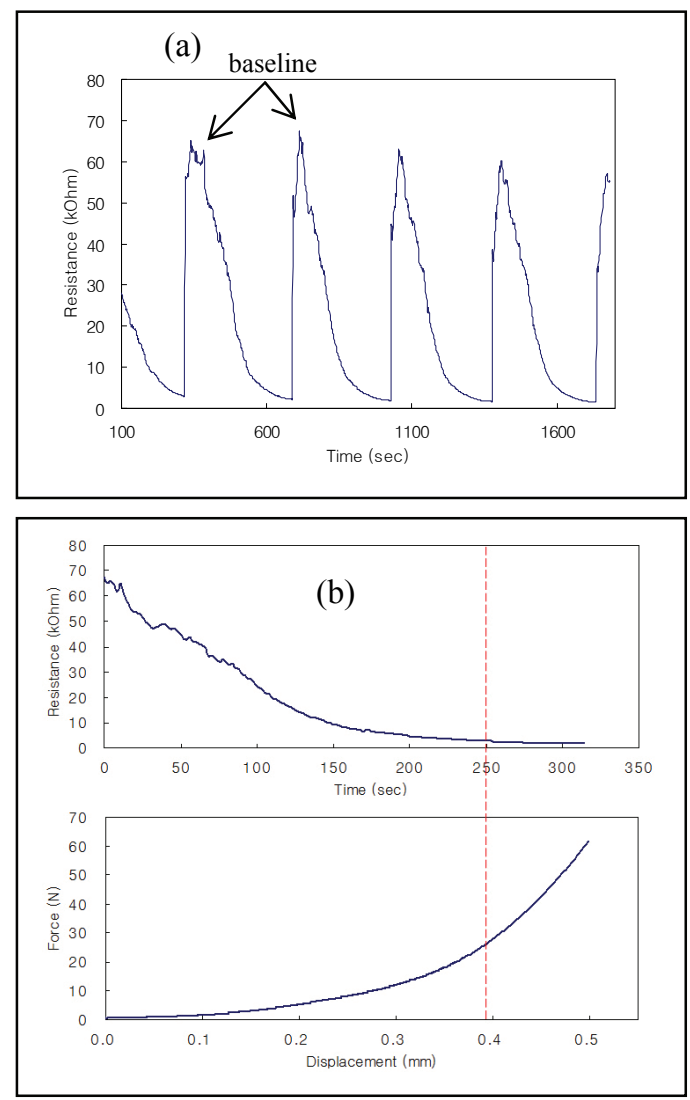

Fig. 7. Zwick compression test of the bare carboncomposite pressure sensor. (a) Resistance vs. time with repeated loading-unloading; (b) simultaneous forcedisplacement and resistance measurement. Test speed: $0.1 \mathrm{~mm} / \mathrm{min}$.

\section{Validation of PMMA cover}

In the final pressure sensing bite-guard design, the pressure sensor needs to be encapsulated by acrylic cover to protect it from the saliva. Hence, the acrylic cover affects the sensitivity of pressure sensor because it upholds most of the applied force. The bending of the acrylic cover induced by the applied load deflects the pressure sensor to result in a different pressure range being sensed. Figure 8 shows the change of resistance from the compression test with $0.87 \mathrm{~mm} \sim 1.20 \mathrm{~mm}$ thick acrylic cover with test jig. 


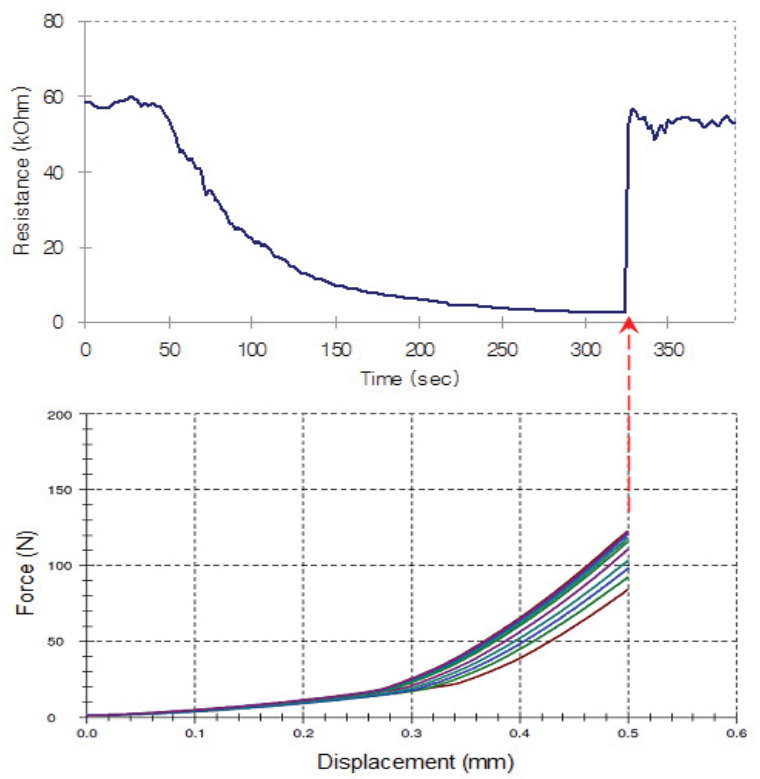

Fig. 8. (a) The change of sensor resistance during (b) load-displacement test of sensor under acrylic cover.

As expected, to achieve specific displacement, higher force was required to bend thicker acrylic cover. As the resistance observed from the bare sensor was a result of compression, therefore, the sensor could serve as a transducer for the displacement of the acrylic cover induced by applied force. Hence, the observed sensitivity would be governed by the modulus of elasticity of the cover material. Based on this, the detection range could be tuned by different cover material. Figure 8 (a) shows that the range of resistance recorded during the compression test was similar to the previous results obtained with bare sensor, but the maximum load at 50\% compression almost doubled to ca. $120 \mathrm{~N}$. The multiple curves shown in Figure 8(b) represent acrylic covers of different thickness.

\section{$\underline{\text { Power Consumption }}$}

The average current draw of the microcontroller at standby mode was $0.76 \mathrm{~mA}$. When the microcontroller was performing $\mathrm{A} / \mathrm{D}$ converting of input signal without wireless transmission, the current draw was $1.1 \mathrm{~mA}$.
During RF transmission, the peak current reached over $11 \mathrm{~mA}$ as shown in Figure 9(a). The capacity of $1.55 \mathrm{~V}$ silver oxide battery (SR43) used in this application was $120 \mathrm{mAh}$. This device was shown to run for over 100 hours continuously at transmission mode. Figure 9(b) shows the voltage drop during a $150 \mathrm{hr}$ continuous (one packet per second) transmission test. Practically, the total duration of bruxism per night is normally less than 30 minutes. Hence, this device could last for several months without changing the battery.

The working range of wireless communication is up to 100 meters line of sight. So the patient could locate the host computer anywhere in the house as this device has a wireless range suitable for most residential environments.
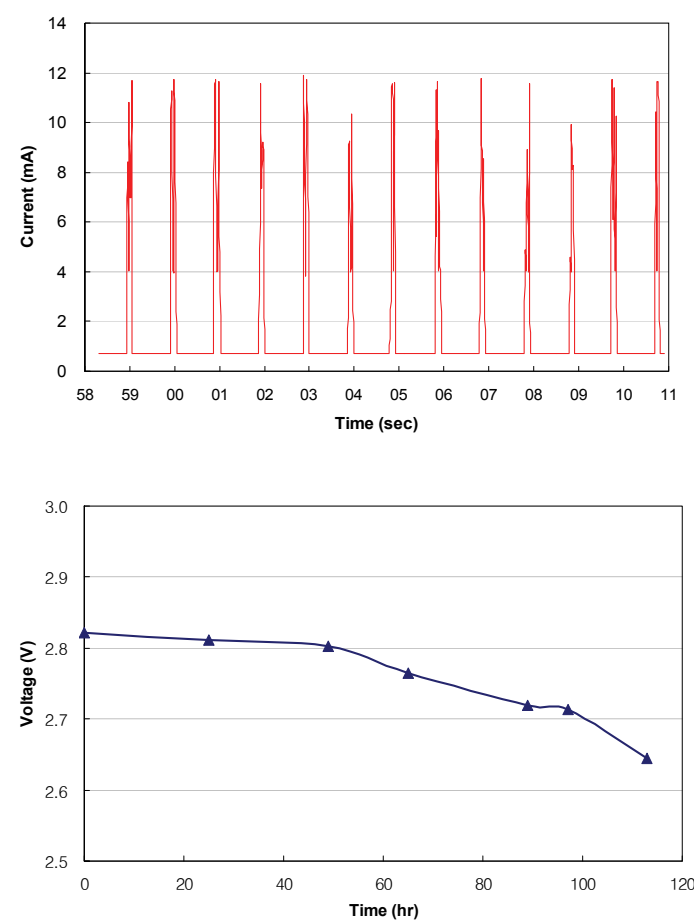

Fig. 9. (a) The current draw of microcontroller in transmission mode: Transmitting frequency $=1 \mathrm{~Hz}(\mathrm{~b})$ Voltage drop of battery during transmission operation.

\section{Calibration of the wireless bruxism monitoring device}

Compression test of the wireless integrated sensing bite- 
guard was performed with Zwick instrument and the A/D value from the sensor was received wirelessly at the PC through the receiver module.
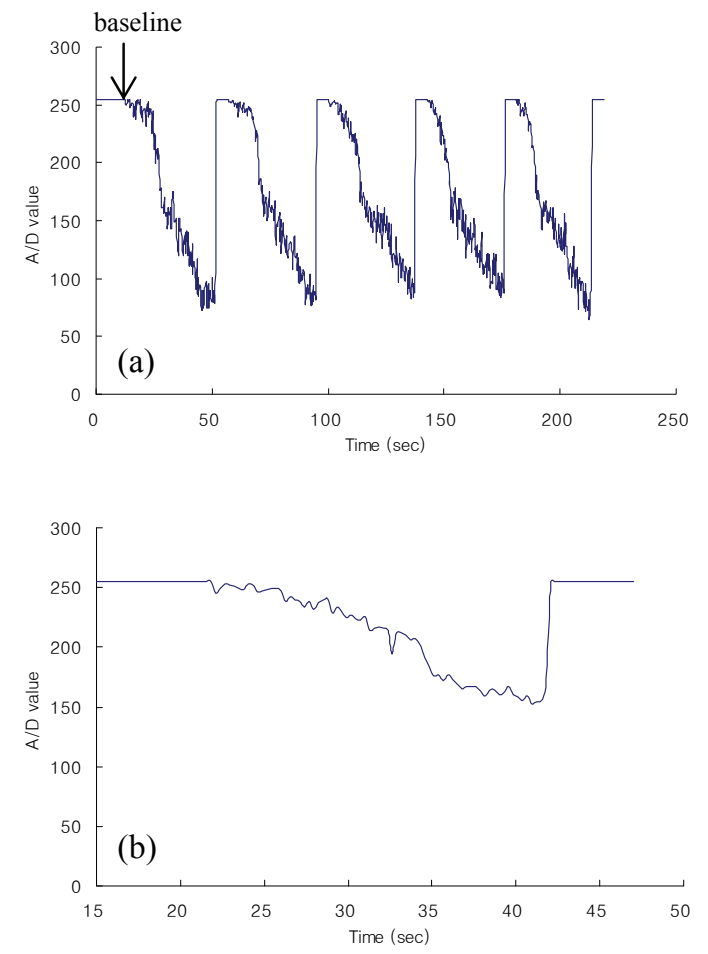

Fig. 10. (a) $A / D$ value from the sensor vs. Time with pressing head at the center of the cover (b) off the center: $4 \mathrm{~mm}$ away from the center of cover.

As shown in Figure 10 (a), good response range between $250-70$ unit was obtained with applied load of 0 to 200 $\mathrm{N}$. Very good reproducibility was also observed from the completed device over 5 consecutive compressing tests with the press head located at the center of the cover. When the pressing position was shifted $4 \mathrm{~mm}$ from the center of the cover, the response range was greatly reduced as shown in Figure 10(b). However, the sensitivity was still sufficient to capture the compression event. In practice, it is not possible to control the point of contact during a tooth grinding event; our data have shown that the proposed device is sensitive enough to capture very mild grinding. As bruxism is characterized by both the force and the duration of the contact; this device is able to record these parameters in real time with high precision. Although we have not perform trials with human at this stage, the results have shown good promise for diagnosing and monitoring bruxism.

\section{Conclusions}

A wireless pressure sensing bite guard has been developed for monitoring the progress of bruxism. The performance of the device appears to be an excellent methodology for diagnosing and monitoring bruxism.

\section{Acknowledgment}

This work is supported by Enterprise Ireland Commercialization Funding grant code: POC-2008-0156.

\section{References}

[1] F. Lobbezoo, J. Van Der Zaag and M. Naeije, "Bruxism: its multiple causes and its effects on dental implants," Journal of Oral Rehabilitation, Vol. 33, pp. 293-300, 2006.

[2] J. Van Der Zaag, F. Lobbezoo, C. M. Visscher, H. L. Hamburger and M. Naeije, "Time-variant nature of sleep bruxism outcome variables using ambulatory polysomnography: implications for recognition and therapy evaluation," Journal of Oral Rehabilitation, Vol. 35, pp. 577-584, 2008.

[3] K. Koyano, Y. Tsukiyama, R. Ichiki and T. Kuwata, "Review Article: Assessment of bruxism in the clinic," Journal of Oral Rehabilitation, Vol. 35, pp. 495-508, 2008.

[4] T. T. T. Dao and G. J. Lavigne, "Oral Splints: The Crutches For Temperomandibular Disorders and Bruxism?," Crit Rev Oral Biol Med, Vol. 9, pp. 345-361, 1998.

[5] T. J. Dylina, “A common sense approach to splint 
therapy," The Journal of Prosthetic Dentistry, Vol. 86, pp. 539-545, 2001

[6] C. R. Macedo, A. B. Silva, M. A. Machado, H. Saconato and G. F. Prado, "Occlusal splints for treating sleep bruxism (tooth grinding)," Cochrane Database of Systematic Reviews, Issue 4, JohnWiley \& Sons, Ltd, pp. $1-23,2008$.

[7] G. Z. Reding, H. Zepelin, J. E. Robinson, S. O. Zimmerman and V. H. Smith, "Nocturnal teeth grinding: all-night psycho-physiologic studies," Journal of Dental Research, Vol. 47, pp. 786-797, 1968.

[8] W. K. Solberg, J. D. Rugh, "The use of biofeedback devices in the treatment of bruxism," Journal of California Dental Association, Vol. 40, pp. 852-853, 1972.

[9] G. J. Lavigne, P. H. Romp, and J. Y. Monstplaisir, "Sleep bruxism: validity of clinical diagnosis criteria in a controlled polysomnographic study," Journal of Dental Research, Vol. 75, pp. 546-552, 1996.

[10] K. Nishigawa, E. Bando and M. Nakano, "Quantitative study of bite force during sleep associated bruxism," Journal of Oral Rehabilitation, Vol. 28, pp. 485-491, 2001.

[11] H. Takeuchi, T. Ikeda and G. T. Clark, "A piezoelectric film-based intrasplint detection method for bruxism," The Journal of Prosthetic Dentistry, Vol. 86, pp. 195-202, 2001

[12] M. Hussain, Y. H. Choa and K. Nihara, "Fabrication process and electrical behavior of novel pressuresensitive composites," Composites: Part A, Vol. 32, pp. 1689-1696, 2001

[13] T. Ding, L. Wang and P. Wang, "Change in electrical resistance of carbon-black-filled silicone rubber composite during compression," Journal of Polymer Science: Part B, Vol. 45, pp. 2700-2706, 2007.

[14] M. Narkis, A. Ram and F. Flasner, "Electrical properties of carbon black filled polyethylene," Polymer Engineering Science, Vol. 18, pp. 649-653, 1978.

[15] K. Miyasaka, K. Watanabe, E. Tojima, H. Aida, M. Sumita and K. Ishikawa, "Electrical conductivity of carbon-polymer composites as a function of carbon contents," Journal of Mater Sci, Vol. 17, pp. 1610-1616, 1982.

[16] S. Choi and J. Jiang, "A novel wearable sensor device with conductive fabric and PVDF film for monitoring cardiorespiratory signal," Sensors and Actuators A, Vol. 128, pp. 317-326, 2006.

[17] C. N. Chien, H. W. Hsu, J. K. Jang, C. L. Rau and F. S. Jaw, "Microcontroller-based wireliee recorder for biomedical signals", Proc. IEEE Engineering in Medicine and Biology Conf., Vol. 5, pp. 5179-5181, 2005. 